\title{
Complexo Littorina ziczac (Gmelin) (Mollusca, Gastropoda, Caenogastropoda) no litoral fluminense: análise morfométrica, distribuição vertical e bioquímica
}

\author{
Ricardo Silva Absalão 1, 2 \\ Renata Alves Pinheiro Roberg ${ }^{1}$
}

\begin{abstract}
The Littorina ziczac (Gmelin) complex (Mollusca, Gastropoda, Caenogastropoda) in the Rio de Janeiro coast: morphometric analysis, vertical distribution and biochemistry. The Littorina ziczac (Gmelin, 1791) species complex was studied in Rio de Janeiro State, southeastern Brazil. The occurrence of three species was confirmed, through the analysis of penis morphology: L. ziczac (Gmelin, 1791), L. lineata d'Orbigny, 1841 and $L$. lineolata d'Orbigny, 1840. There is a correlation between the morphology of the penis and shell shape, thus it was possible to make a discrimination model based in conchologic data, with an efficiency of $75 \%$. However, this model cannot be applied to others samples that weren't included in its formulation, due to phenotypic plasticity of the shell caused by biotic and abiotic factors. In the 9 areas studied $L$. lineolata was always the most abundant species. No vertical stratification in the distribution of the species was verified. From the nine enzymatic systems examined, only Pgi, Pgm e Mpi, presented identifiable patterns and no diagnostic locus was identified in any species. Nevertheless, S and I suggest the individualization of $L$. ziczac and $L$. lineata, respectively.

KEY WORDS. Littorina, species complex, vertical distribution, biochemistry, Rio de Janeiro, Brazil
\end{abstract}

Os litorinídeos são caenogastrópodes que habitam, tipicamente, os substratos consolidados da zona entre marés em todo o mundo e, particularmente, a zona supralitoral. No Brasil se distribuem do Cabo Orange ao Chuí (RIos 1994), desde que o substrato apropriado esteja presente.

Apesar da ampla distribuição e da abundância numérica dos litorinídeos, eles não têm sido bem estudados no Brasil, com exceção de MARCUS \& MARCUS (1963). Por outro lado, em escala mundial, são relativamente abundantes os estudos sobre sua taxonomia, ecologia e biologia.

Um problema comum que parte considerável desses estudos tem enfrentado é o de espécies crípticas ou, como é comumente chamado entre os malacologistas, um complexo de espécies. Um complexo de espécies deve ser entendido como a existência de duas ou mais espécies com características semelhantes e geralmente associados a um habitat ou conjunto de habitats. Assim, pode-se esperar dificuldades relacionadas à identificação das espécies integrantes de complexos desta natureza.

1) Departamento de Zoologia, Instituto de Biologia, Universidade Federal do Rio de Janeiro. 21941-570 Rio de Janeiro, Rio de Janeiro, Brasil.

2) Departamento de Biologia Animal e Vegetal, Instituto de Biologia, Universidade do Estado do Rio de Janeiro. Rua São Francisco Xavier 524, 20550-900 Rio de Janeiro, Rio de Janeiro, Brasil. 
Atualmente continuam a ocorrer controvérsias quanto a identificação das espécies dentro dos complexos: LINDBERG (1982), BECK (1986), ROTH \& PRESSLEY (1986), VERDUIN (1986) e PALMER et al. (1990).

Embora a existência de complexos não seja exclusiva do gênero Littorina Ferussac, 1822, neste tem recebido considerável atenção o complexo de $L$. saxatilis (HANNAFORD ELLIS 1983, 1984; JANSON 1983, 1985; JOHANNASSEN \& JOHANNASSEN 1990; KNIGHT \& WARD 1991) e o complexo de L. scutulata (Gould, 1849) (MURRAY 1982; MASTRO et al. 1982).

Os métodos mais utilizados para estudar as espécies crípticas do gênero Littorina são: 1) análise estatística a partir de dados morfométricos (GOODWIN \& FISH 1977; VERMEIJ 1982; MOYSE et al. 1982; MURRAY 1982; JANSON \& WARD 1984; JANSON 1985; SUNDBERG 1988; GRAHAME \& MiLl 1989; JOHANNASSEN \& JOHANNASSEN 1990; KNIGHT \& WARD 1991; WARWICK \& ClARKE 1991); 2) estudos bioquímicos, especialmente utilizando-se a eletroforese (WARD \& WARDWICK 1980; MOYSE et al. 1982; MASTRO et al. 1982; JASON 1985, 1987; JANSON \& Ward 1984; SeEley 1986; FeVolden \& Garner 1987; Mill \& Grahame 1988; WARD 1990; JOHANNASSEN \& JOHANNASSEN 1990; KNIGHT \& WARD 1991); e 3) características fisiológicas e/ou anatômicas dos indivíduos (GOODWIN \& FisH 1977; HANNAFORD ELLIS 1983; BANDEL \& KADOLSKY 1982; RAFFAELLI 1982; MURRAY 1982; MASTRO et al. 1982; MOYSE et al. 1982; JANSON 1985, 1987; SeEley 1986; Grahame \& Mill 1986, 1989; SundBerg 1988).

O complexo Littorina ziczac foi caracterizado como tal, pela primeira vez por BORKOWSKI \& BORKOWSKI (1969) para a Florida (EUA). De acordo com esses autores o complexo é constituído por três espécies: Littorina ziczac (Gmelin, 1791), Littorina lineolata d'Orbigny, 1840 e Littorina lineata d'Orbigny, 1841 que, segundo eles, podem ser discriminadas a partir de evidências biológicas, ecológicas e morfológicas.

O complexo Littorina ziczac tem como limites de distribuição o sul do Uruguai e o norte das Bermudas; variando sua distribuição vertical conforme a região considerada, contudo as espécies sempre se apresentariam estratificadas dentro da zona supralitoral (BORKOWSKI \& BORKOWSKI 1969).

MARCUS \& MARCUS (1963) foram os primeiros a estudar o que se conhecia como L. ziczac no Brasil e, a não ser pelos trabalhos de Rios (1970, 1975, 1985), de âmbito muito mais geral, só em 1983, Luiz Eduardo Lima (com. pessoal) veio confirmar a ocorrência do complexo no litoral fluminense; no entanto, afirmou não ter encontrado nenhuma estratificação vertical entre as espécies integrantes, muito embora não apresentasse resultados que corroborassem sua posição. Além disso, segundo Luiz Eduardo Lima (com. pessoal) seria necessário dissecar os animais para identificá-los pois os caracteres conquiliológicos apresentados por ele eram excessivamente frágeis.

Assim, motivados em parte pela questão da aparente ausência de estratificação vertical entre as espécies do complexo em oposto a BORKOWSKI \& BORKOWSKI (1969), e em parte, pela necessidade de se discriminar seus integrantes sem a necessidade de dissecá-los -essencial ao desenvolvimento de quaisquer trabalhos 
ecológicos que requeiram uma identificação dos animais vivos - resolveu-se desenvolver este estudo que tem por objetivos: 1) discriminar as espécies do complexo Littorina ziczac, baseando-se em características morfométricas, que pudessem ser utilizadas em campo; 2) verificar a possível ocorrência de estratificação vertical entre as espécies do complexo no litoral do estado do Rio de Janeiro.

\section{MATERIAL E MÉTODOS}

\section{Distribuição Vertical}

As coletas de material para determinar os padrões de distribuição vertical foram realizadas com "quadrats" de $200 \mathrm{~cm}^{2}$ em dois transectos perpendiculares à linha d'água. Os "quadrats" foram posicionados a cada $10 \mathrm{~cm}$ de deslocamento vertical e de modo que a partir do início da ocorrência dos litorinídeos todos os níveis de maré (de $10 \mathrm{em} 10 \mathrm{~cm}$ ) fossem amostrados. Todos os indivíduos presentes no interior dos "quadrats" foram coletados, fixados e conservados em álcool $70 \%$. Em laboratório foram separados por sexo e os machos classificados de acordo com as características morfológicas da genitália; logo, os dados de distribuição e abundância referem-se ao número médio de indivíduos machos das amostras. Para esta finalidade coligiram-se amostras das seguintes localidades e anos: Arpoador (1989), Prainha (1989) e Urca (1990) no município do Rio de Janeiro; Praia Brava (1989) e Forno (1990) em Arraial do Cabo; Praia do Forte (1990), das Conchas (1990) em Cabo Frio; Boa Viagem (1989) e Itaipu (1989) em Niterói. Durante estas coletas, virtualmente todas as inclinações dos costões foram representadas.

Baseou-se amplamente nas descrições das genitálias masculinas para assinalar cada indivíduo como pertencente a um dos táxons do complexo; contudo, denominou-se, como um quarto táxon, Littorina af. lineolata em virtude da semelhança entre a genitália desta e a de L. lineolata (Fig. 1).

Este material encontra-se depositado na coleção malacológica do Departamento de Zoologia, Instituto de Biologia, Universidade Federal do Rio de Janeiro (UFRJ), lotes: 2754, 2777, 2778, 2797, 2798, 2800 a 2806, 2812 a 2821, 2824 a $2827,3172,3710^{*}$ a $3714^{*}$ (* lotes utilizados para realização dos desenhos em câmara clara, ver a seguir).

\section{Análise Morfométrica}

Para a análise conquiliológica separou-se 30 indivíduos machos de cada táxon. Esses animais tiveram suas conchas individualmente desenhadas em câmara clara e a partir desses desenhos, foram obtidas as seguintes medidas: comprimento total (CT), largura total (LT), comprimento do último anfracto (CUA), comprimento da abertura (CA), largura da abertura (LA), ângulo externo (AE), ângulo interno (AI) e ângulo de inserção (AIN) (Fig. 2).

A fim de minimizar uma possível influência do tamanho dos indivíduos na análise, trabalhou-se com medidas proporcionais: $\mathrm{V}_{1}=\mathrm{CT} / \mathrm{LT}, \mathrm{V}_{2}=\mathrm{CT} / \mathrm{CUA}, \mathrm{V}_{3}$ $=\mathrm{CUA} / \mathrm{LA}, \mathrm{V}_{4}=\mathrm{CA} / \mathrm{LA}, \mathrm{V}_{5}=\mathrm{LT} / \mathrm{LA}$ e $\mathrm{V}_{6}=\mathrm{AE} / \mathrm{AI}$. Através de $\mathrm{V}_{1}$ tem-se uma idéia da forma da concha, se é mais bojuda ou afilada; $V_{2}$ indica o quanto da concha é ocupada pelo último anfracto, onde a perda de água seria potencialmente maior. 


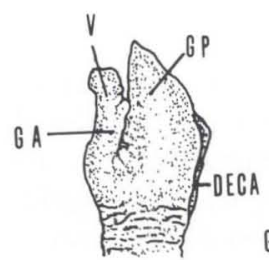

3

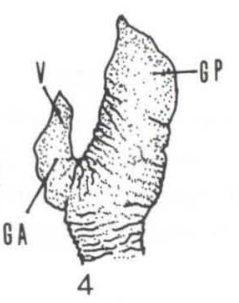

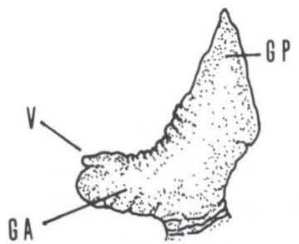

1

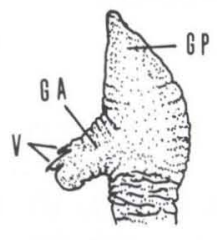

2
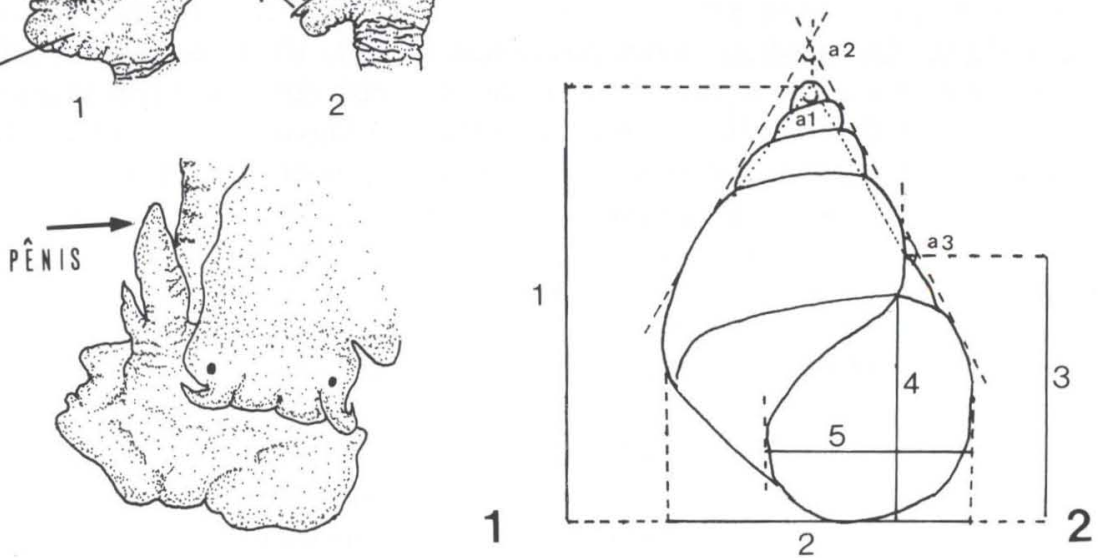

Figs 1-2. (1) Genitália masculina dos táxons componentes do complexo Littorina ziczac: (GP) glândula peniana, (V) verruga, (GA) glândula acessória, (DECA) ducto espermático de calha aberta, (1) L. lineolata, (2) L. af. lineolata, (3) L. ziczac, (4) L. lineata, (2) Representação esquemática da concha de um espécime do complexo Littorina ziczac e as medidas utilizadas na análise morfométrica: (1) comprimento total, (2) largura total, (3) comprimento do último anfracto, (4) comprimento da abertura, (5) largura da abertura, (a1) ângulo interno da espira, (a2) ângulo externo da espira, (a3) ângulo de inserção.

As variáveis 3 e $4\left(\mathrm{~V}_{3}\right.$ e $\mathrm{V}_{4}$ respectivamente) referem-se à abertura, fornecendo noções de sua forma - alongada ou alargada -conseqüentemente indica se a superfície de contato com o ar é grande ou pequena. Essa informação é complementada por $\mathrm{V}_{5}$, que mostra o quanto da largura da concha é ocupada pela abertura. A $V_{6}$ representa o grau de convexidade das voltas da concha. Concluindo, as variáveis de 1 a 6 juntas, indicam a forma a concha (inclusive a da abertura) que, em última instância, tem significado ecológico para o animal.

Além dessas, utilizou-se, também, os valores absolutos do ângulo interno (AI), do ângulo externo (AE), do ângulo de inserção (AIN), do número de bandas claras na abertura (BAN) e do número de linhas em $1 \mathrm{~mm}$ da concha (LIN).

Estes dados foram submetidos à análise discriminante multivariada do pacote estatístico SPSS, no Núcleo de Computação Eletrônica (UFRJ). Essa técnica obtém a melhor combinação linear de variáveis para discriminar, o mais eficiente- 
mente, entre grupos definidos "a priori". Os grupos definidos "a priori" foram aqueles identificados a partir da genitália. Para detalhes da técnica consulte-se LEGENDRE \& LEGENDRE (1983).

Para testar a aplicabilidade do modelo discriminante, utilizou-se indivíduos coletados em localidades diferentes daquelas nas quais foram obtidos os indivíduos utilizados para a construção do modelo. Estas localidades foram Ibicuí e Praia Brava (Mangaratiba, Rio de Janeiro), Camburí (Vitória, Espírito Santo), Saquarema e Grumari (Rio de Janeiro).

\section{Análise de genética bioquímica - eletroforese}

Para esta finalidade foram realizadas novas coletas nas praias do Arpoador, Vermelha, Joatinga e Ilha Rasa (Rio de Janeiro), Itaipu (Niterói), Prainha e Pontal do Atalaia (Arraial do Cabo) e Porto Galo (Angra dos Reis) (todas realizadas em 1992). Para evitar que as características bioquímicas destes animais fossem adulteradas, as dissecações foram realizadas com os animais vivos, os machos separados e mantidos congelados a $-18^{\circ} \mathrm{C}$ por um período inferior a 30 dias. Por fim selecionou-se 10 espécimens de Littorina lineolata, 11 de L. lineata, 06 de L. af. lineolata e 04 de L. ziczac.

A análise em si, empregou o processo eletroforético, tendo como base de análise as isoenzimas destes animais. Para a obtenção do homogeneizado para análise, cada indivíduo foi macerado inteiro com água destilada em diluição de 1:1. O gel suporte da eletroforese consistiu em uma solução à $12,5 \%$ de amido e o tempo de corrida foi de seis horas com tampão triscítrico, $\mathrm{pH} 8$ (TC 8 ), à $4^{\circ} \mathrm{C}$, e sob potência entre 3600-4200 mW. A voltagem utilizada foi de 120 volts e a corrente de 30-35 mA. Os detalhes da técnica podem ser obtidos em SolÉ-CAVA \& THORPE (1987). Foram testados nove sistemas de ensimas: leucina aminopeptidase (Lap), alfa esterase ( $\alpha$-Est), lactato desidrogenase (LDH), malato desidrogenase (Mdh), manose fosfato isomerase (Mpi), fosfoglucose isomerase (Pgi), fosfoglucomutase (Pgm), xantina oxidase (Xod) e isocitrato desigrogenase (Idh). Dessas, apenas Pgi, Pgm e Mpi apresentaram padrões interpretáveis.

Calculou-se as similaridades entre os táxons através do índice de Thorpe (S) e de Ney (I) (THORPE 1983). O índice de Thorpe é calculado a partir da soma das menores sobreposições. E para o cálculo do índice de Ney (I) usa-se a fórmula:

$$
I=\frac{\sum\left(f_{i} \times f_{j}\right)}{\sqrt{\sum f_{i}^{2} \times \sum f_{j}^{2}}}
$$

sendo: ( $\left.f_{i}\right)$ freqüência gênica de $A,\left(f_{j}\right)$ freqüência gênica de $B$

\section{RESULTADOS}

\section{Distribuição Vertical}

As figuras 3-5 constituem-se numa amostra gráfica da distribuição vertical dos táxons. Pode-se notar que não há separação vertical entre eles, e sim uma ampla sobreposição em todos os níveis do costão. Além disto, não detectou-se alternância significativa da forma dominante de um costão para outro. Na maioria deles o táxon 
mais abundante foi aquele considerado como Littorina lineolata. Por este motivo elegeu-se, apenas, estes três costões como representativos dos demais para fins de apresentação nesse trabalho.
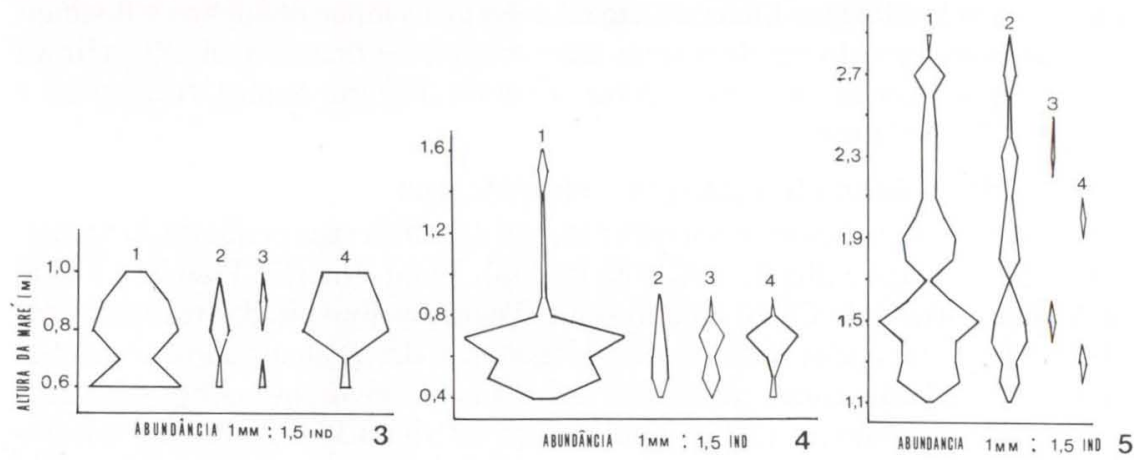

Figs 3-5. Distribuição vertical e abundância dos táxons do complexo Littorina ziczac, no estado do Rio de Janeiro. (3) Praia da Urca; (4) Praia do Forno, Arraial do Cabo; (5) Praia de Boa Viagem, Niterói. Os números referem-se aos indivíduos machos dos seguintes táxons: (1) L. lineolata, (2) L. af. lineolata, (3) L. ziczac e (4) L. lineata.

\section{Análise Morfométrica}

A figura 6 apresenta a posição relativa de cada táxon em relação ao plano criado pelas funções discriminantes:

$\mathrm{F} 1=11,23 \mathrm{~V}_{1}+4,02 \mathrm{~V}_{2}+5,89 \mathrm{~V}_{5}+8,14 \mathrm{~V}_{6}+0,01 \mathrm{AIN}+0,28 \mathrm{AI}+0,02$ $\mathrm{LIN}-0,72 \mathrm{~V}_{3}-6,36 \mathrm{~V}_{4}-0,1 \mathrm{AE}-0,13 \mathrm{BAN}-44,93$ e F2 $=0,002 \mathrm{~V}_{1}+6,24 \mathrm{~V}_{2}$ $+0,74 \mathrm{~V}_{4}+11,13 \mathrm{~V}_{5}+1,03 \mathrm{~V}_{6}+0,01 \mathrm{AIN}+0,02 \mathrm{AI}+0,53 \mathrm{LIN}-3,5 \mathrm{~V}_{3}-0,02$ $\mathrm{AE}-0,12 \mathrm{BAN}-29,34$.

Pode-se observar uma considerável discriminação entre $L$. lineolata, $L$. $z i c z a c$ e o conjunto formado por $L$. lineata e $L$. af. lineolata que não se separaram com clareza entre si.

A percentagem de discriminação correta, isto é, de concordância entre a classificação baseada na morfologia da genitália masculina e a baseada nas medidas morfométricas das conhas foi de $60,83 \%$ (Tab. I). Devido a "proximidade" observada entre $L$. lineata e $L$. af. lineolata, refez-se a análise considerando-se as duas formas como um único táxon conquiliológico.

O mapa territorial (Fig. 7) obtido a partir das novas funções discriminantes em função da recombinação dos táxons:

$\mathrm{F} 1=12,19 \mathrm{~V}_{1}+3,43 \mathrm{~V}_{2}+6,84 \mathrm{~V}_{5}+8,47 \mathrm{~V}_{6}+0,01 \mathrm{AIN}+0,28 \mathrm{AI}+0,29$ LIN $-1,89 \mathrm{~V}_{3}-6,34 \mathrm{~V}_{4}-0,1 \mathrm{AE}-0,17 \mathrm{BAN}-45,57$ e F2 $=5,11 \mathrm{~V}_{1}+1,97 \mathrm{~V}_{2}$ $+1,08 \mathrm{~V}_{4}+15,37 \mathrm{~V}_{5}+2,49 \mathrm{~V}_{6}+0,01 \mathrm{AIN}+0,02 \mathrm{AI}+0,53 \mathrm{LIN}-10,08 \mathrm{~V}_{3}-$ $0,02 \mathrm{AE}-0,38 \mathrm{BAN}-28,11$ mostrou boa discriminação dos três táxons e, como resultado final, obteve-se um percentual de discriminação correta de 75,83\% (Tab. II). 


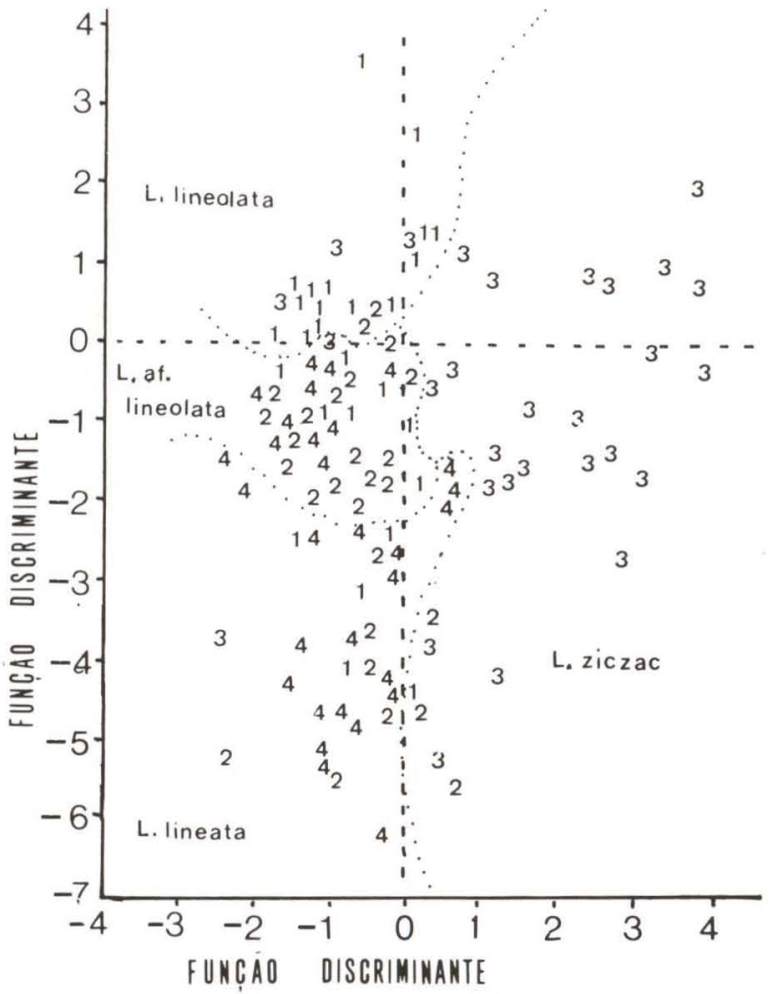

Fig. 6. Mapa territorial resultante da análise discriminante multivariada com os quatro táxons. Os números referem-se aos indivíduos machos dos seguintes táxons: (1) L. lineolata, (2) L. af. lineolata, (3) L. ziczac e (4) L. lineata.

Tabela I. Demonstrativo do percentual de discriminação do modelo conquiliológico com quatro táxons.

\begin{tabular}{lcccc}
\hline \multicolumn{1}{c}{ Táxon } & L. lineolata & L. af.lineolata & L. ziczac & L. lineata \\
\hline L. lineolata & 66,70 & 16,70 & 0,00 & 16,70 \\
L. af.lineolata & 10,00 & 50,00 & 6,70 & 33,30 \\
L. ziczac & 13,30 & 6,70 & 66,70 & 13,30 \\
L. lineata & 6,70 & 33,30 & 6,70 & 60,00 \\
\hline
\end{tabular}

Percentual geral de discriminação correta: $60,83 \%$

Tabela II. Demonstrativo do percentual de discriminação do modelo conquiliológico após recombinação dos táxons.

\begin{tabular}{lccc}
\hline \multicolumn{1}{c}{ Táxon } & L. lineolata & L. af.lineolata + L. lineata & \multicolumn{1}{c}{ L. ziczac } \\
\hline L. lineolata & 73,33 & 23,33 & 3,33 \\
L. af.lineolata + L. lineata & 18,33 & 78,33 & 3,33 \\
L. ziczac & 13,33 & 13,33 & 73,33 \\
\hline
\end{tabular}

Percentual geral de discriminação correta: $75,83 \%$ 


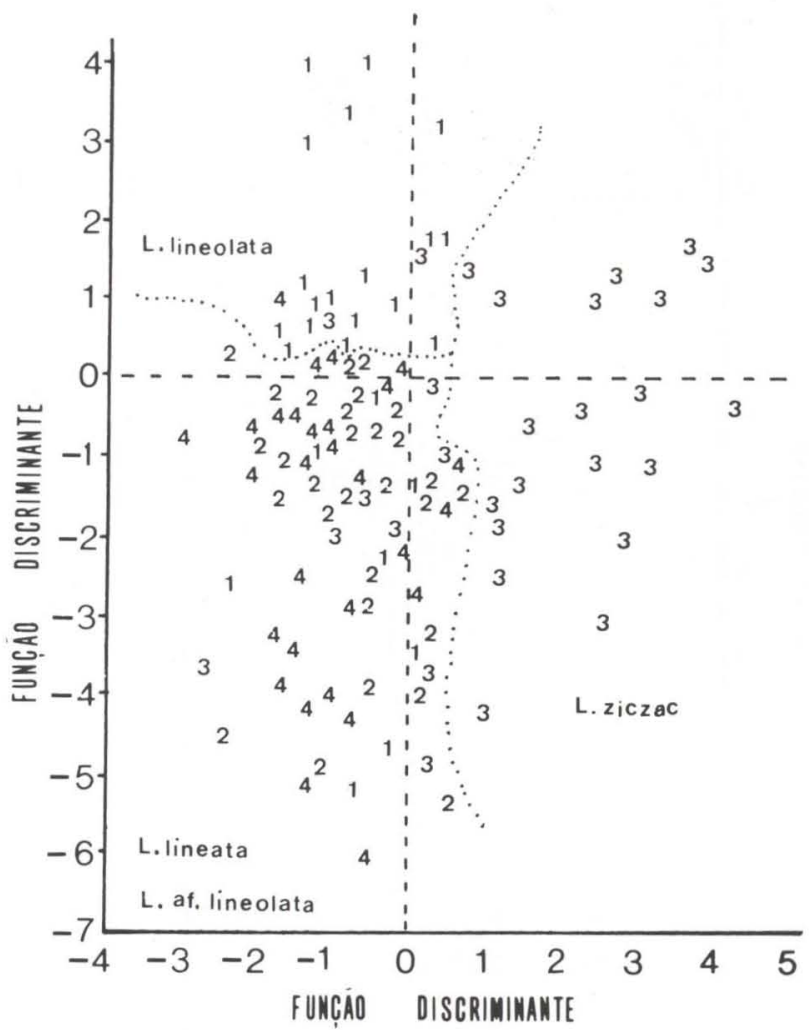

Fig. 7. Mapa territorial resultante da análise discriminante multivariada após a recombinação dos quatro táxons em três (Littorina af. lineolata e L. lineata consideradas em conjunto). Os números referem-se aos indivíduos machos dos seguintes táxons: (1) L. lineolata, (2) L. af. lineolata, (3) L. ziczac e (4) L. lineata.

Utilizando-se os dados morfométricos dos indivíduos machos provenientes de localidades outras que não aquelas que forneceram os espécimens para as análises precedentes, constatou-se uma concordância muito baixa entre os indivíduos provenientes das novas amostras e as previsões baseadas nas amostras iniciais (Tabs III, IV). Em outras palavras, houve uma baixa concordância entre a classificação proposta pelo modelo (baseada na morfologia da concha) e a baseada na morfologia da genitália masculina destes "novos" espécimens.

\section{Análise de genética bioquímica - eletroforese}

Para cada sistema enzimático foram calculadạs as freqüências genotípicas e gênicas e a seguir calcularam-se, também, as similaridades entre os táxons pelo índice de Thorpe (Tab. V) e pelo índice de Ney (Tab. VI). Segundo ThorPe (1983) valores de $\mathrm{S}$ acima de 0,9 indicam indivíduos coespecíficos; valores entre $0,85 \mathrm{e}$ 0,35 indivíduos cogenéricos e valores abaixo de 0,3 são supragenéricos. 
Tabela III. Teste do modelo de discriminação conquiliológico com material proveniente de outras localidades (análise com quatro táxons). (E) Erro, (A) acerto, (\%) percentagem de acerto, (total) total de indivíduos utilizados.

\begin{tabular}{|c|c|c|c|c|c|c|c|c|c|c|c|c|c|c|c|}
\hline \multirow{2}{*}{$\begin{array}{c}\text { Local } \\
\text { Táxon/situação }\end{array}$} & \multicolumn{3}{|c|}{ Ibicui } & \multicolumn{3}{|c|}{ Praia Brava } & \multicolumn{3}{|c|}{ Grumari } & \multicolumn{3}{|c|}{ Camburi } & \multicolumn{3}{|c|}{ Saquarema } \\
\hline & $E$ & A & $\%$ & $E$ & A & $\%$ & $E$ & $A$ & $\%$ & $E$ & A & $\%$ & $E$ & A & $\%$ \\
\hline L. lineolata & 31 & 2 & 6 & 6 & 2 & 25 & 43 & 8 & 15 & 25 & 10 & 28 & 11 & 2 & 18 \\
\hline L. af.lineolata & 10 & 1 & 9 & 1 & 0 & 0 & 3 & 0 & 0 & 6 & 0 & 0 & 1 & 1 & 50 \\
\hline L. ziczac & 0 & 0 & 0 & 0 & 0 & 0 & 0 & 0 & 0 & 1 & 0 & 0 & 0 & 1 & 100 \\
\hline L. lineata & 0 & 0 & 0 & 0 & 0 & 0 & 1 & 0 & 0 & 1 & 0 & 0 & - & - & - \\
\hline Total & 41 & 3 & 7 & 7 & 2 & 22 & 47 & 8 & 14 & 33 & 10 & 23 & 12 & 4 & 25 \\
\hline
\end{tabular}

Tabela IV. Teste do modelo de discriminação conquiliológico com material proveniente de outras localidades (análise com três táxons). (E) Erro, (A) acerto, (\%) percentagem de acerto, (total) total de individuos utilizados.

\begin{tabular}{|c|c|c|c|c|c|c|c|c|c|c|c|c|c|c|c|}
\hline \multirow{2}{*}{$\begin{array}{c}\text { Local } \\
\text { Táxon/situaçăo }\end{array}$} & \multicolumn{3}{|c|}{ Ibicui } & \multicolumn{3}{|c|}{ Praia Brava } & \multicolumn{3}{|c|}{ Grumari } & \multicolumn{3}{|c|}{ Camburi } & \multicolumn{3}{|c|}{ Saquarema } \\
\hline & $E$ & A & $\%$ & $E$ & A & $\%$ & $E$ & A & $\%$ & $E$ & A & $\%$ & $E$ & A & $\%$ \\
\hline L. lineolata & 33 & 0 & 0 & 8 & 0 & 0 & 51 & 0 & 0 & 35 & 0 & 0 & 13 & 0 & 0 \\
\hline $\begin{array}{l}\text { L. af.lineolata + } \\
\text { L. lineata }\end{array}$ & 9 & 2 & 18 & 1 & 0 & 0 & 4 & 0 & 0 & 7 & 0 & 0 & 2 & 0 & 0 \\
\hline L. ziczac & 0 & 0 & 0 & 0 & 0 & 0 & 0 & 0 & 0 & 0 & 0 & 0 & 0 & 1 & 100 \\
\hline Total & 42 & 2 & 5 & 9 & 0 & 0 & 55 & 0 & 0 & 42 & 1 & 2 & 15 & 1 & 6 \\
\hline
\end{tabular}

Assim, segundo o índice de Thorpe, pode-se afirmar que L. ziczac individualiza-se com clareza de $L$. lineata e de $L$. lineolata, enquanto que os táxons restantes não se individualizam entre si (Tab. V). Enquanto que o índice de Ney (Tab. VI) reforça a separação de L. lineata dos outros membros do complexo e os demais não se individualizariam entre si. Ressalte-se que não se encontrou evidências a favor do "status" de espécie para o morfo denominado L. af. lineolata através de nenhum dos dois índices.

Tabela V. Proximidade filogenética dos táxons do complexo Littorina ziczac calculada a partir do indice de Thorpe utilizando-se os sistemas enzimáticos Pgi, Pgm e Mpi.

\begin{tabular}{|c|c|c|c|}
\hline \multicolumn{4}{|c|}{ Sistema Enzimático Pgi } \\
\hline$S(L$. lineolata - L. af, lineolata $)$ & $=0,93$ & $\mathrm{~S}($ L. af. lineolata - L. ziczac $)$ & $=0,92$ \\
\hline S (L. lineolata - L. ziczac) & $=0,85$ & $S(L$. af. lineolata $-L$. lineata) & $=0,94$ \\
\hline $\mathrm{S}(\mathrm{L}$. lineolata - L. lineata $)$ & $=0,99$ & $S(L$. ziczac - L. lineata $)$ & $=0,86$ \\
\hline \multicolumn{4}{|c|}{ Sistema Enzimático Pgm } \\
\hline S (L. lineolata - L. af. lineolata) & $=0,75$ & $\mathrm{~S}($ L. af. lineolata - L. ziczac) & $=0,96$ \\
\hline S (L. lineolata - L. ziczac) & $=0,75$ & $S(L$. af. lineolata - L. lineata) & $=0,80$ \\
\hline$S(L$. lineolata $-L$. lineata $)$ & $=0,95$ & $\mathrm{~S}(\mathrm{~L}$. ziczac - L. lineata $)$ & $=0,80$ \\
\hline \multicolumn{4}{|c|}{ Sistema Enzimático Mpi } \\
\hline$S$ (L. lineolata - L. af. lineolata) & $=0,93$ & $S(L$. af. lineolata - L. ziczac) & $=0,83$ \\
\hline$S(L$. lineolata - L. ziczac $)$ & $=0,90$ & $S(L$. af. lineolata $-L$. lineata) & $=0,96$ \\
\hline$S(L$. lineolata - L. lineata $)$ & $=0,92$ & $\mathrm{~S}(\mathrm{~L}$. ziczac - L. lineata $)$ & $=0,82$ \\
\hline \multicolumn{4}{|c|}{ Média } \\
\hline $\mathrm{S}($ L. lineolata - L. af. lineolata $)$ & $=0,87$ & $\mathrm{~S}($ L. af. lineolata - L. ziczac $)$ & $=0,90$ \\
\hline S (L. lineolata - L. ziczac) & $=0,83$ & $S(L$. af. lineolata $-L$. lineata) & $=0,90$ \\
\hline$S(L$. lineolata - L. lineata $)$ & $=0,95$ & $S(L$. ziczac - L. lineata $)$ & $=0,83$ \\
\hline
\end{tabular}


Tabela VI. Proximidade filogenética dos táxons do complexo Littorina ziczac calculada a partir do índice de Ney utilizando-se os sistemas enzimáticos Pgi, Pgm e Mpi.

\begin{tabular}{|c|c|c|c|}
\hline \multicolumn{4}{|c|}{ Sistema Enzimático Pgi } \\
\hline I (L. lineolata - L. af. lineolata $)$ & $=0,99$ & I (L. af. lineolata - L. ziczac) & $=1,00$ \\
\hline I (L. lineolata - L. ziczac) & $=0,98$ & I ( L. af. lineolata $-L$. lineata $)$ & $=0,25$ \\
\hline I (L. lineolata - L. lineata) & $=0,44$ & I (L. ziczac - L. lineata $)$ & $=0,16$ \\
\hline \multicolumn{4}{|c|}{ Sistema Enzimático Pgm } \\
\hline$I(L$. lineolata $-L$. af. lineolata $)$ & $=0,93$ & I (L. af. lineolata - L. ziczac) & $=0,99$ \\
\hline I (L. lineolata - L. ziczac) & $=0,94$ & I (L. af. lineolata - L. lineata) & $=0,94$ \\
\hline I (L. lineolata - L. lineata $)$ & $=0,99$ & I (L. ziczac - L. lineata) & $=0,95$ \\
\hline \multicolumn{4}{|c|}{ Sistema Enzimático Mpi } \\
\hline I (L. lineolata - L. af. lineolata) & $=0,99$ & I (L. af. lineolata - L. ziczac) & $=0,97$ \\
\hline I (L. lineolata - L. ziczac) & $=1,00$ & I (L. af. lineolata - L. lineata) & $=0,99$ \\
\hline I (L. lineolata $-L$. lineata $)$ & $=0,99$ & I (L. ziczac - L. lineata $)$ & $=0,98$ \\
\hline \multicolumn{4}{|c|}{ Média } \\
\hline$I(L$. lineolata $-L$. af. lineolata $)$ & $=0,97$ & I (L. af. lineolata - L. ziczac) & $=0,98$ \\
\hline I (L. lineolata - L. ziczac) & $=0,97$ & I (L. af. lineolata - L. lineata $)$ & $=0,72$ \\
\hline I (L. lineolata - L. lineata $)$ & $=0,80$ & I (L. ziczac- - L. lineata $)$ & $=0,69$ \\
\hline
\end{tabular}

\section{DISCUSSÃO}

MARCUS \& MARCUS (1963) não consideraram L. lineolata para o Brasil. FLores (1973) propôs a ocorrência das três espécies no Brasil. Além disso, BORKOWSKI \& BORKOWISKI (1969) citam que L. ziczac brasiliensis (VERMEIJ \& PORTER 1971) provavelmente representaria L. lineata.

Luiz Eduardo Lima (com. pessoal) explica a ausência de L. lineolata nos sensos faunísticos realizados no Brasil como resultado da confusão entre $L$. lineolata e $L$. ziczac. Segundo esse mesmo autor, L. lineolata apresenta um pênis com uma glândula acessória na base e possui uma banda clara na abertura da concha. Para $L$. lineata, ele descreve uma concha com duas bandas claras na abertura e pênis com uma glândula acessória constituída de um lobo glandular com uma verruga na extremidade. Em relação à $L$. ziczac, afirma não haver glândula acessória no pênis e que MARCUS \& MARCUS (1963) equivocaram-se ao identificar tal estrutura, que seria, segundo ele, uma dobra terminal do lóbulo de fixação. Sustenta, ainda, que o material estudado por ele difere do apresentado por outros autores (BEQUAERT 1943; BORKOWSKI \& BORKOWSKI 1969; RIOS 1975). Nesse estudo considerou-se, com base na morfologia, a presença de uma glândula acessória com uma pequena verruga como característico de L. ziczac (Fig. 1). Em relação ao número de bandas claras presentes na abertura da concha, não se obteve qualquer padrão que pudesse separar os táxons, em oposto ao encontrado por BORKOWSKI \& BORKOWSKI (1969) e BORKOWSKI (1975).

\section{Distribuição Vertical}

Não se observou a existência de um padrão de distribuição vertical estratificado para as espécies do complexo Littorina ziczac nas localidades estudadas. Por outro lado, BORKOWSKI \& BORKOWSKI (1969) detectaram uma distribuição tipicamente estratificada com $L$. lineata mais afastada da linha d'água, $L$. lineolata nos 
níveis intermediários e $L$. ziczac mais próxima a linha dágua. Talvez a distribuição vertical das espécies do complexo varie em conformidade com a latitude, apresentando-se estratificada no seu limite de ocorrência setentrional e não estratificada no meridional.

\section{Análise Morfométrica}

A análise discriminante multivariada sobre as variáveis conquilio-morfométricas resultou num modelo discriminante de razoável eficiência, mas aparentemente aplicável, apenas, as amostras que serviram de base a sua construção (Tabs IV, V). As variações relativamente amplas nas variáveis conquiliológicas que, em última instância invalidaram a aplicação do modelo construído a outras amostras alheias àquelas originalmente utilizadas na elaboração do mesmo, podem representar respostas adaptativas às alterações nas condições ambientais como reportado por Claudia Magalhães (com. pessoal) para o complexo L. ziczac que alcançaram maiores tamanhos em localidades mais expostas a ação das ondas e por GooDWIN \& FISH (1977) que comprovaram haver relação entre o grau de exposição do local e as medidas da concha como altura, largura e altura da abertura de $L$. obtusata e $L$. mariae. Além desses, JANSON (1983) demonstrou que L. saxatilis é apta a apresentar fenótipos distintos conforme ocupe locais com diferentes graus de exposição, conclusão idêntica a que chegou MCMAHON \& YIPP (1992) trabalhando com Nodilittorina unifasciata. Fatores biológicos como a presença de predadores (JANSON 1983), de estarem em alopatria ou simpatria com outras espécies do complexo que integram (GraHAME \& Mill 1989; GraHAME et al. 1992) e efeitos da densidade são, também, fatores importantes na expressão da variabilidade conquiliológica entre os litorinídeos e que também poderiam estar contribuindo a pobre capacidade de discriminação desse modelo quando aplicado a outras amostras. $\mathrm{Na}$ verdade estudos que encontraram evidências de uma base genética para as diferenças populacionais utilizaram espécies com desenvolvimento direto e ausência de uma fase larvar amplamente dispersável. Nessas espécies a morfologia da concha poderia estar sujeita a pressões de seleção em escala microambiental o que levaria ao desenvolvimento de raças genéticas com formas de concha distintas em populações isoladas (CROTHERS 1985). Por outro lado, em espécies com fases larvares livres, ou com cápsulas ovígeras como nas espécies do complexo Littorina ziczac (BORKOWSKI \& BORKOWSKI 1969) que garantam o fluxo gênico entre populações, as variações conquiliológicas seriam muito mais reflexo da plasticidade fenotípica que de diferenças genéticas (MCMAHON \& YIPP 1992; GrAHAME et al. 1992).

BORKOWSKI \& BORKOWSKI (1969) utilizaram-se de características conquiliológicas para separar as três espécies do complexo $L$. ziczac na Flórida (EUA). BORKOWSKI (1975) afirma que estas mesmas "características conquiliológicas" seriam inadequadas à separação dos táxons integrantes do complexo L. ziczac, mas as utiliza em indivíduos adultos na distinção entre $L$. lineata e L. lineolata. BORKOWSKI (1975) afirma que há grande confusão nas determinações das três espécies do complexo L. ziczac realizadas por outros autores como por exemplo a de L. jamaicensis C.B. Adams, 1850 realizada por BANDEL (1974) para o que seria realmente $L$. lineata d'Orbigny, 1841 ou ainda a identificação equivocada de $L$. lineolata como L. lineata realizada pelo mesmo autor. Dada a possível influência 
de fatores bióticos e abióticos na determinação do fenótipo final das espécies do complexo $L$. ziczac em cada localidade não é surpreendente a confusão reinante entre os conquiliologistas. Sem dúvidas, a observação anatômica das genitálias continuará como um instrumento decisivo para o reconhecimento das espécies integrantes do complexo L. ziczac.

\section{Análise Bioquímica}

A análise eletroforética não foi capaz de encontrar loci diagnósticos, o que pode-se levar a considerar a possibilidade de que se trata de uma única espécie com um alto grau de polimorfismo em relação a morfologia da genitália masculina. WARD (1990) ao desenvolver abordagem similar a apresentada neste trabalho também não encontrou locus diagóstico algum entre Littorina saxatilis e L. arcana; entretanto, manteve o status de espécies para ambas baseado nas diferenças da freqüência alélica e no número de loci polimórficos. MiLL \& GRAHAME (1988) haviam conseguido separá-las através do sistema enzimático esterase (Est.) além de diferenciarem as fêmeas maduras pela morfologia e cor da genitália. JOHANNESSON \& JOHANNESSON (1990) afirmaram que $L$. arcana e $L$. saxatilis seriam espécies crípticas e que por conta disso apresentariam diferenças em aloenzimas muito pequenas, mas que o fluxo gênico entre indivíduos pertencentes a mesma espécie, em locais distintos, seria maior do que aquele entre indivíduos de ambas as espécies presentes em um mesmo local. KNIGHT \& WARD (1991) argumentaram que a falta de loci diagnósticos e a baixa distância gênica entre elas seria devido a recente especiação a partir de um ancestral comum.

Em nosso caso, a análise eletroforética não foi conclusiva (ausência de locus diagnóstico) apesar do valor do índice de Ney se constituir em forte evidência a favor da separação de L. lineata dos táxons restantes. Por outro lado, o índice de Thorpe também sugere a individualização de $L$. ziczac dos outros táxons. Infelizmente o pequeno número de indivíduos examinados e, principalmente, de sistemas enzimáticos considerados recomendam extrema cautela na interpretação da ausência de um locus disgnóstico, o que poderia em parte, explicar os resultados não concordantes entre os indices de Ney e de Thorpe.

Se, por um lado, a presença de um único locus é determinante na validação da separação de dois táxons quaisquer, por outro lado sua ausência não significa que estejamos tratando com uma única entidade biológica polimórfica, como demonstrado no caso do complexo L. saxatilis. O complexo L. ziczac demanda, ainda, considerável esforço em vários campos de pesquisa antes que uma posição definitiva possa ser proposta.

\section{CONCLUSÕES}

Existe considerável associação entre a morfologia da genitália masculina das espécies integrantes do complexo Littorina ziczac e variáveis conquiliológicas que traduzem a forma das conchas. Esta associação genitália-forma da concha é restrita as amostras investigadas, ou seja, não pode ser extrapolada para populações não estudadas quando da construção de um modelo discriminante. 
Os táxons do complexo não se apresentaram verticalmente estratificados nos costões estudados.

A análise eletroforética não foi conclusiva quanto ao reconhecimento específico das espécies integrantes do complexo L. ziczac; no entanto o índice de Ney aponta para a individualização de $L$. lineata e o índice de Thorpe para a de $L$. ziczac dos táxons restantes.

\section{REFERÊNCIAS BIBLIOGRÁFICAS}

BANDEL, K. 1974. Studies on Littorinidae from the Atlantic. Veliger 17 (2): 92-114. BANDEL, K. \& D. KADOLSKY. 1982. Western atlantic species of Nodilittorina (Gastropoda: Prosobranchia): Comparative morphology and its functional, ecological, philogenetic and taxonomic implications. Veliger 25 (1): 11-42.

BECK, L. 1986. The radula, shell and soft body of thetwin species Gibbula umbilicalis (Da Costa) and Gibbula pennanti (Philippi) (Gastropoda: Prosobranchia, Trochidae). Arch. Mollusckenkd 117 (1-3): 11-18.

BEQUAERT, J.C. 1943. The genus Littorina in the Western Atlantic. Johnsonia 7: $1-28$.

Borkowski, T.V. 1975. Variability among Caribbean Littorinidae. Veliger 171 (4): 369-377.

BORKOWSKI, T.V.\& M.R. BoRKOWSKI. 1969. The ziczac species complex. Veliger 11 (4): 408-414.

CROTHERS, J.H. 1985.Two different patterns of shell-shape variation in the dogwhelk Nucella lapillus (L. ). Biol. Jour. Linn. Soc. 25 (4): 339-353.

FELVODEN, S.E. \& S.P. GARDINER. 1987. Environmental stress and allozyme variation in Littorina littorea (Prosobranchia). Mar. Ecol. 39: 129-136.

FLORES, C. 1973. La familia Linidae (Mollusca: Mesogastropoda) en las aguas costeras de Venezuela. Bol. Inst. oceanogr. Cumana 12 (1): 3-22.

GoodWIN, B.J. \& J.D. FISH. 1977. Inter- and intra- specific variation in Littorina obtusata and L. marie (Gastropoda: Prosobranchia). Jour. Moll. Stud. 43: 241-254.

GRAHAME, J. \& P.J. MiLL. 1986. Relative size of the foot of two species of Littorina on a rocky shore in Wales. Jour. Zool. 208: 229-236.

. 1989. Shell shape variation in Littorina saxatilis and L. arcana: a case of character displacement? Jour. Mar. Biol. Ass. U.K. 69: 837-855.

Grahame, J.; P.J. Mill; M. Double \& S.L. Hull. 1992. Patterns of variation in Aat-1 allele frequencies in rough periwinkles (Littorina) suggest similar selection regimes rather than conspecificity. Jour. Mar. Biol. Ass. U.K. 72 (2): 499-502.

HANNAFORD Ellis, C. 1983. Patterns of reproduction in four Littorina species. Jour. Moll. Stud. 49: 98-106.

1984. Ontogenetic change in colour shell pattern in Littorina negleta Bean (1844). Jour. Conch. 31: 343-347.

JASON, K. 1983. Selection and migration in two distinct phenotypes of Littorina saxatilis in Sweden. Oecologia 59: 58-61.

. 1985. A morphologic and genetic analysis of Littorina saxatilis 
(Prosobranchia) from Venice, and on the problem of saxatilis-rudis nomenclature. Biol. Jour. Lin. Soc. 24: 51-59.

. 1987. Allozyme and shell variation in two marine snails (Littorina, Prosobranchia) with different dispersal abilities. Biol. Jour. Lin. Soc. 30: 245-256.

JASON, K. \& R.D. WARD. 1984. Microgeographic variation in allozyme and shell characters in Littorina saxatilis Olivi (Prosobranchia, Littorinidae). Biol. Jour. Lin. Soc. 22: 289-307.

JOHANNESSON, K. \& B.O. JOHANNESSON. 1990. Genetic variation with Littorina saxatilis (olivi) and Littorina neglecta Bean: Is L. neglecta a good species? Hidrobiologia 193: 89-97.

KNIGHT, A.J. \& R.D. WARD. 1991. The genetic relationships of three taxa in the Littorina saxatilis species complex (Prosobranchia: Littorinidae). Jour. Moll. Stud. 57: 81-91.

LEGENDRE, L. \& P. LEGENDRE. 1983. Numerical ecology. New York, Elsevier Sci. Publ., 419p.

LINDBERG, D.R. 1982. A multivariate study of morphological variation of the limpet Notoacmea depicta and its synonyms Notoacmea gabatella and Notoacmea lepisma (Gastropoda: Acaeidae). Bull. South Calif. Acad. Sci. 81: 87-96.

MARCUS, E. \& E.B.R. MARCUS. 1963. Mesogastropoden von der Küste von São Paulo. Abhandlungen der Mathematisch-Naturwissenschaftlichen Klasse 1: $1-105$.

MASTRO, E.; V. CHOW \& D. HEDGECOCK. 1982. Littorina scutulata and Littorina plena: Sibling species status of two prosobranch gastropod species confirmed by eletrophoresis. Veliger 24 (3): 239-246.

MCMAHON, R. \& M.W. YIPP. 1992. Evaluation of evaporative cooling as a mechanism for regulation of tissue temperatures during insolation in twelve species of Hong Kong mangrove and rocky shore prosobranchs gastropods, p.745-767. In: B. MOUTON (Ed.). The marine flora and fauna of Hong Kong and Southern China. Vol. 2: Introduction taxonomy and ecology. Hong Kong, Univ Press.

Mill, P.J. \& J. Grahame. 1988. Esterase variability in the gastropods Littorina saxatilis (Olivi) and $L$. arcana Ellis. Jour. Moll. Stud. 54: 347-353.

MOYSE, J.; J.P. THORPE \& E. AL-HAMADANI. 1982. The status of Littorina aestuarii Jeffreys an approach using morphology and biochemical genetics. Jour. Conch. 31: 7-15.

MURRAY, T. 1982. Morphological characterization of the Littorina scutulata speceis complex. Veliger 24 (3): 233-238.

PALMER, A.R.; S.D. GAYRON \& D.S.W. DrufF. 1990. Reprodutive, morphological and genetic evidence for two cryptic species of Northeastern Pacific Nucella. Veliger 33 (4): 325-338.

RAFFAELLI, D. 1982. Recent ecological research on some european species of Littorina. Jour. Moll. Stud. 48: 342-354.

Rios, E.C. 1970. Coastal Brazilian Seashells. Rio Grande, Museu Oceanográfico do Rio Grande, 255p.

. 1975. Brazilian Marine Mollusks. Rio Grande, Museu Oceanográfico 
Fundação Universidade de Rio Grande, 331p.

— 1985. Seashells of Brazil. Rio Grande, Museu Oceanográfico Fundação Universidade de Rio Grande, 328pp.

Roth, B. \& P.H. PRESSLEY. 1986. Observations on the range and natural history of Manadenia setosa (Gastropoda: Pulmonata) in the Klamath Montains, California (USA) and the taxonomy of the same relates species. Veliger 29 (2): 169-182.

SEELEY, R.H. 1986. Intense natural selection caused a rapid morphological transition in a living marine snail. Proc. Nat. Acad. Sci. USA 83: 6897-6901.

SOlÉ-CAVA, A. \& J.P. THORPE. 1987. The use of eletrophoresis in sponge taxonomy, p.243-258. In: J. VAcelet \& N. BouRy Esnault (Eds). The Sponge Taxonomy. Nato Asi Series Vol. 13, Springer Verlag.

SUNDBERG, P. 1988. Microgeographic variation in shell characters of Littorina saxatilis Olivi-a question mainly of size ? Biol. Jour. Lin. Soc. 35: 169-184.

THORPE, J.P. 1983. Enzime variations, genetic distance and evolutionary devergence in relation to levels of taxonomic seoaration, p. 131-152. In: Protein polymorphism: Adaptative and taxonomic significance. Systematic Association.

Verduin, A. 1986. On the systematics of some recent Rissoa. Basteria 50: 13-24.

VERMEIJ, G.J. 1982. Environmental change and evolutionary history of the periwinkle (Littorina littorea) in North America. Evolution 36: 561-580.

VERMEIJ, G.J. \& J.W. PORTER. 1971. Some characteristics of the dominant intertidal molluscs from rocky shores in Pernambuco, Brazil. Bull. Mar. Sci. 21 (2): 440-454.

WARD, R.D. 1990. Biochemical genetic variation in the genus Littorina (Prosobranchia: Mollusca). Hydrobiologia 193: 53-69.

WARD, R.D. \& T. WARWICK. 1980. Genetic diferentiation in the molluscan species Littorina rudis and Littorina arcana (Prosobranchia: Littorinidae). Biol. Jour. Lin. Soc. 14: 417-428.

WARWICK, R.M. \& K.R. CLARKE. 1991. A comparison of some methods for analysing changes in benthic community structure. Jour. Mar. Biol. Ass. U.K. 71: $225-244$.

Recebido em 31.X.1997; aceito em 22.III.1999. 